\title{
THE GPS SATELLITE AND PAYLOAD
}

\author{
Andrew Codik and Robert A. Gronlund \\ Rockwell International Corporation \\ Satellite Systems Division \\ 12214 Lakewood Boulevard \\ Downey, California, USA 90241
}

\begin{abstract}
The NAVSTAR/Global Positioning System Satellite is briefly outlined as is the technique for navigation using signals from such satellites. A description of the GPS navigation subsystem with emphasis on its microprocessor and its interfaces and software routines is given.
\end{abstract}

\section{INTRODUCTION}

The NAVSTAR/Global Positioning System (GPS) satellite segment consists of a number of satellites in 12 hour orbits at an altitude of $20,183 \mathrm{Km}$ (10,898 nautical miles). The first NAVSTAR satellite was launched in February 1978, and the second in May 1978. The full constellation will consist of 24 satellites and will provide any user on or near Earth simultaneous visibility of at least 6 satellites.

Navigation signals are transmitted by each satellite on two L-band frequencies $(1575 \mathrm{MHz}$ and $1227 \mathrm{MHz}$ ) to permit corrections to be made for ionospheric delays in signal propagation time. These signals are modulated by two pseudorandom noise codes: $\mathrm{P}$, which provides for precision measurements of pseudorange, and C/A, which provides for easy acquisition of desired satellites. Data, consisting of satellite ephemerides, time correction coefficients and other useful information to the users, is modulo two added to the pseudorandom codes.

The satellite L-band antenna radiates near uniform power to the users. Using signals received from four satellites a user can make a position fix; within 10 meters in latitude, longitude and altitude. The amount of time required for a position fix depends on the sophistication of the receiving system and can vary from tens of seconds to several minutes; the receiver can also provide the user vehicles velocity. 


\section{NAVIGATION TECHNIQUE}

A user can determine his position in three dimensions if he can receive L-band signals from four satellites. The precision of a user's determination is a function of the geometry of the satellites used. Almanac information transmitted from the satellites is available to the user for automatic or manual selection of the four satellites which present the best geometry and therefore allow the highest precision in position determination.

Performance of the navigation function requires synchronization of satellite clocks with GPS system time. An atomic frequency standard in each satellite monitored by the GPS Control Segment and clock correction data are provided to each satellite by the Control Segment for transmission to users as digital correction coefficients. A user need not have a precise frequency standard if he utilizes signals from four satellites, the fourth satellite permits estimation of the user's frequency standard bias. The four user position equations contain four unknowns, three dimensions and the user's frequency standard bias, and are subject to simultaneous solution.

\section{PHASE I SATELLITE}

The GPS NAVSTAR satellites which are in orbit or being readied for launch are 3 axis stabilized with the navigation subsystem antenna pointing toward Earth. The satellite tracks the sun about the yaw axis, allowing the solar array panels to have a single degree of freedom. This results in a beneficial thermal environment within the satellite. One side of the satellite is exposed to the sun, and two sides always face deep space. Thermal control of the satellite is simplified, the major heat producing components are mounted on the deep space exposed sides, and control is accomplished by means of louvers and thermostatically controlled heaters. Figure 1 is an exploded view of the Phase I Satellite and lists the salient features of the major satellite subsystems.

The Telemetry, Tracking and Command (TT\&C) subsystem is fully compatible with the Air Force Space Ground Link System and also provides the uplink capability that is used by the GPS Upload Station located at Vandenberg Air Force Base. The Upload Station transmits to the satellite the ephemerides and other data for use by the Navigation Subsystem's Processor. The TT\&C subsystem antennas provide omnidirectional coverage since they are used for commanding and telemetry transmission during all phases of the mission, including those during which the navigation subsystem antenna and forward TT\&C antennas are not pointed at Earth. 


\section{SATELLITE MISSION PAYLOAD}

The mission payload of the NAVSTAR satellites is the Navigation Subsystem. It consists of:

- Frequency Standards - 3 Rubidium atomic standards are provided, they are used in a dormant redundancy configuration and are selected by ground command.

- Processor/Baseband - It generates the pseudorandom codes and modulates them with digital data; it is internally doubly redundant and sections can be switched by command.

- The RF Equipment, consisting of carrier frequency synthesizers, modulotor/ intermediate power amplifiers and high power amplifiers. The two L-band carriers are combined in a diplexer/filter which in turn feeds the shaped beam antenna. The RF equipment is totally redundant and individually switchable except for the diplexer and the antenna.

The Navigation Subsystem configuration is shown in Figure 2, and is representative of the first 3 NAVSTAR satellites. An additional cesium atomic frequency standard will be added to satellites number 4 and subsequent during Phase I.

\section{RF Section}

The navigation subsystem RF section, as shown in Figure 2, derives its signals from the 10.23 MHz output of the frequency standard. Two L-band carriers denoted $\mathrm{L}_{1}$ and $\mathrm{L}_{2}$ are synthesized from this standard frequency. The $\mathrm{L}_{1}$ carrier, which has a frequency of 1575.42 MHz is simultaneously modulated by the $10.23 \mathrm{MHz}$ precision $(\mathrm{P})$ pseudorandom noise (PRN) code and the $1.023 \mathrm{MHz}$ acquisition (C/A) PRN code in phase quadrature. The $\mathrm{L}_{2}$ carrier, at $1227.6 \mathrm{MHz}$, is modulated by either (selection is by ground command) the $\mathrm{P}$ code or the-C/A code. The $\mathrm{P}$ and C/A PRN codes are then exclusive-ored with the 50 bit per second navigation data. The L-band carriers are amplified by the intermediate power amplifiers (IPA) and high power amplifiers (HPA). The $\mathrm{L}_{1}$ HPA includes an incremental high power mode, which, when selected by command from the ground, results in an increased output of the C/A component.

\section{Antenna}

The intent of the NAVSTAR antenna is to provide equal power density to all terrestrial users. This is accomplished with a shaped beam antenna: a quad helix encircled by a ring of eight helices. Figure 3 shows the antenna layout and the theoretical antenna pattern components and resultant pattern. The actual measured antenna pattern is shown in Figure 4. 
The combined results of the actual antenna pattern and the predicted RF section outputs are plotted as a function of user antenna elevation angle and satellite antenna aspect angle, and are shown in Figure 5. Also shown are the specification requirements, which are the bases for user receiver design. The significant signal margins of the predicted results have been verified by measurements of NAVSTAR I AND NAVSTAR 2 using the Camp Parks, California, Air Force Facility.

\section{Frequency Standard}

The frequency standard is the heart of the Navigation Subsystem because it is the source of the GPS ranging function. The frequency standard-short term and long-term stability characteristics and predictability determine the basic navigation accuracy that can be achieved by the Global Positioning System; an uncertainty of one nanosecond in clock (or code) phase is equivalent to approximately one foot of range uncertainty.

Figure 6 shows the regions of acceptable and unacceptable stability on an Allen Variance plot, for the GPS Phase I frequency standard. Also shown in Figure 6 are actual laboratory measurements of Allen Variance for several production rubidium frequency standards used on NAVSTAR satellites. Note that the actual frequency standard performance is better than the specification requirement by approximately an order of magnitude. This frequency standard stability translates into potentially higher accuracy of position determination by GPS users. The frequency standard frequency is adjustable $b$ ground command. The phase difference (integral of frequency difference) is adjusted by either changing the code state of the baseband and/or modifying the clock correction coefficients transmitted in the Navigation Data message.

\section{Digital Section}

The digital section of the NAVSTAR satellite navigation subsystem consists of the Processor/Baseband Assembly which includes 3 processors, each assembled on a two sided circuit board, and three sets of baseband electronics, each assembled on two boards.

The baseband electronics generates the $10.23 \mathrm{MHz}$ precision $(\mathrm{P})$ code and the $1.023 \mathrm{MHz}$ acquisition (C/A) code and modulo two adds to these codes the 50 bits per second navigation data formatted by the navigation processor. Also, the baseband electronics produces the 19 bit time of week count (or Z-count) which repeats in seven days and increments one binary count every 1.5 seconds. This Z-count is transferred to the processor every 1.5 seconds. The baseband electronics also has the capability of aligning the $\mathrm{P}$ code and Z-count with respect to a ground based system standard via a command message transferred from the processor. 
The primary function of the navigation processor is storage, formatting and outputting of the navigation data that are modulo two added to the PRN codes and transmitted via the L-band signals. Other functions of the navigation processor include:

a. Initializing (bootstrap) the processor.

b. Receipt and storage of navigation data and control data from ground upload stations.

c. Verification of the validity of uploads and in case of errors in the uploads notifying the Control Segment via telemetry. The telemetry information is formatted for either L-band or S-band transmission or for both.

d. Performance of diagnostics of processor hardware and dumps of processor memory contents via the L-band RF links, and the S-band SGLS telemetry link.

\section{Processor Hardware}

The navigation processor consists of a PPS-4 microprocessor augmented by an Arithmetic Logic Unit (ALU) with interrupt capability, as shown in Figure 7. The ALU and its Random Access Memory (RAM) used as a buffer/scratch pad, provide the upload interface with the 1000 bit-per-second SGLS S-band command link. The ALU RAM stores the uploaded data, as received from the ground, and performs a series of simple checks. Both the GPS Control Segment's Upload Station and the AF Satellite Control Facility's Remote Tracking Stations have the ability to communicate with the processor. The Central Processing Unit, which performs most of the processing functions and the ALU interface with the input/output (I/O) devices and with the program and data memory via the instruction/data bus and the address bus. The program memory consists of three 2 $\mathrm{K} \times 8$ bit read only memories (ROM); the data memory consists of $244 \mathrm{~K}$ x 1 bit dynamic NMOS RAM'S.

\section{Processor Firmware}

The navigation processor program uses a combination of interrupt driven logic and polled discretes and flags to initiate the tasks which must be performed on a continuous basis. Figure 8 is a top level flow diagram of the GPS On-Board Computer Program (OBCP). When power is applied to the processor it goes into its bootstrap routine which initializes the processor memory to a state of readiness for initialization of all other routines as the interrupt, discretes, or flags are set. Without further commands or uploads from the ground the processor outputs a nominal navigation data stream and SGLS Telemetry data.

During data uploading from the Control Segment or the AFSCF, an interrupt occurs once per millisecond on the average; the nominal upload rate is 1000 baud, consisting of 1,0, and $\mathrm{S}$ (space) pulses. Following the processing of each pulse, after the interrupt, the processor returns to the interrupted routine and continues processing. The upload message 
is blocked as shown in Figure 9. Each word consists of sixteen bits, followed by an odd parity bit. Three types of data blocks are used: (1) the address block, a one time 32 bit word, that provides access to the processor, (2) a series of data blocks, of 250 words or less, with a checksum incorporated and (3) an end-of-message word that closes the access to the processor. The individual data blocks are inbedded in a stream of S-pulses.

Each data block, as shown in Figure 10, contains information on the type of data that is being transmitted, the location where the data is to be stored and identifying information.

By using both row parity and columnary checksum computations a very low rate of undetected bit error rate can be achieved, specifically the estimated equivalent BER for a block is $\sim 3 \times 10^{-19}$.

Following completion of upload of a block of data, the Primary Data Upload routines compare the resulting checksum with the uploaded value and if the upload is error free these routines output a verifacation code for transmission via L-bond and S-band telemetry and transfer the data to the main processor memory. Should errors be detected during the upload process, the processor aborts further processing of the upload block, and outputs the appropriate telemetry code for transmission.

The output of navigation data from the processor to the baseband electronics is accomplished under control of tables which determine the format and content of the navigation data frame. The Navigation Data routines offer broad flexibility in the format and content of the navigation message, which can vary from one subfrome of 300 bits up to 8 subframes of 2400 bits. Figure 11 is an example of a typical navigation data frame, presently in use on the Phase I system. The example shows a five subframe frame. Another aspect of the flexibility available to the Control Segment is the capability to select the numbers of times each navigation data output block is repeated, that is, how long a given set of ephemerides and/or clock correction data are made available to users. The navigation data includes 6 bits of Hamming parity in each 30 bit data word, for validation of the data by the users. The 6 bits of parity are assigned by the ground (prior to uploading) for the data portion of the message, the processor computes and assigns parity for the telemetry word (TLM) and the Handover word (HOW).

The SGLS Telemetry data routine is coiled when the satellite PCM telemetry subsystem strobes the processor. This routine makes available to the PCM telemetry subsystem the most recent upload status or diagnostic or memory dump information. The output rate is eight words per second or one word per second. 
The Read Z-Counter routine transfers the Z-count, the 19 bit count of GPS time, from the baseband electronics to the processor every 1.5 seconds. This count repeats every seven days and is used by the processor in several ways:

a) The 17 most significant bits are output in the second word of each navigation data subframe.

b) The 3 least significant bits are used in the maintenance of navigation data output synchronization.

c) All 19 bits are used to time the performance of events including initiation of new navigation data frame formats, code and time alignments, diagnostics and memory dumps and are stored in a designated location upon occurrence of an attitude control subsystem roll momentum dump.

d) The fourth through eleventh most significant bits are used to time tag the occurrence of satellite attitude control subsystem roll momentum dumps.

The Address Key Upload routine provides the AFSCF the capability for changing the content of a protected set of memory locations, in the processor main memory, in which primary upload address keys are stored. The Alternate Data Upload routine provides the AFSCF the capability of a low rote upload path via the command decoder.

The Roll Momentum Dump Report routine outputs, via the L-band links, the time of occurrence of roll momentum dumps.

The Code/Time Alignment Message routine transfers an 88 bit command message to the baseband electronics which will change the Z-count and/or P-code output by the baseband, as commanded by the Control Segment. The realignment can be as small as one chip ( 100 nanoseconds) or up to one week in length.

The Diagnostic and RAM Dump routines provide for diagnostics of the CPU logic, the ALU and CPU RAMS and for dumps of the content of the ALU and CPU RAM. Results of these diagnostics and dumps may be via the TLM word in the navigation data subframe or via SGLS telemetry.

The Upload Address Verification routine formulates any of several upload status messages which inform the GPS Control Segment or AFSCF of errors in or success of uploads.

\section{SUMMARY}

The Phase I GPS satellites and ground segments have shown the feasibility of the GPS concept. The navigation accuracy and operational flexibility of the system are being tested. Among the contributors to this operational flexibility testing are the satellite subsystems including the navigation subsystem, switchable redundancy and the processor. 


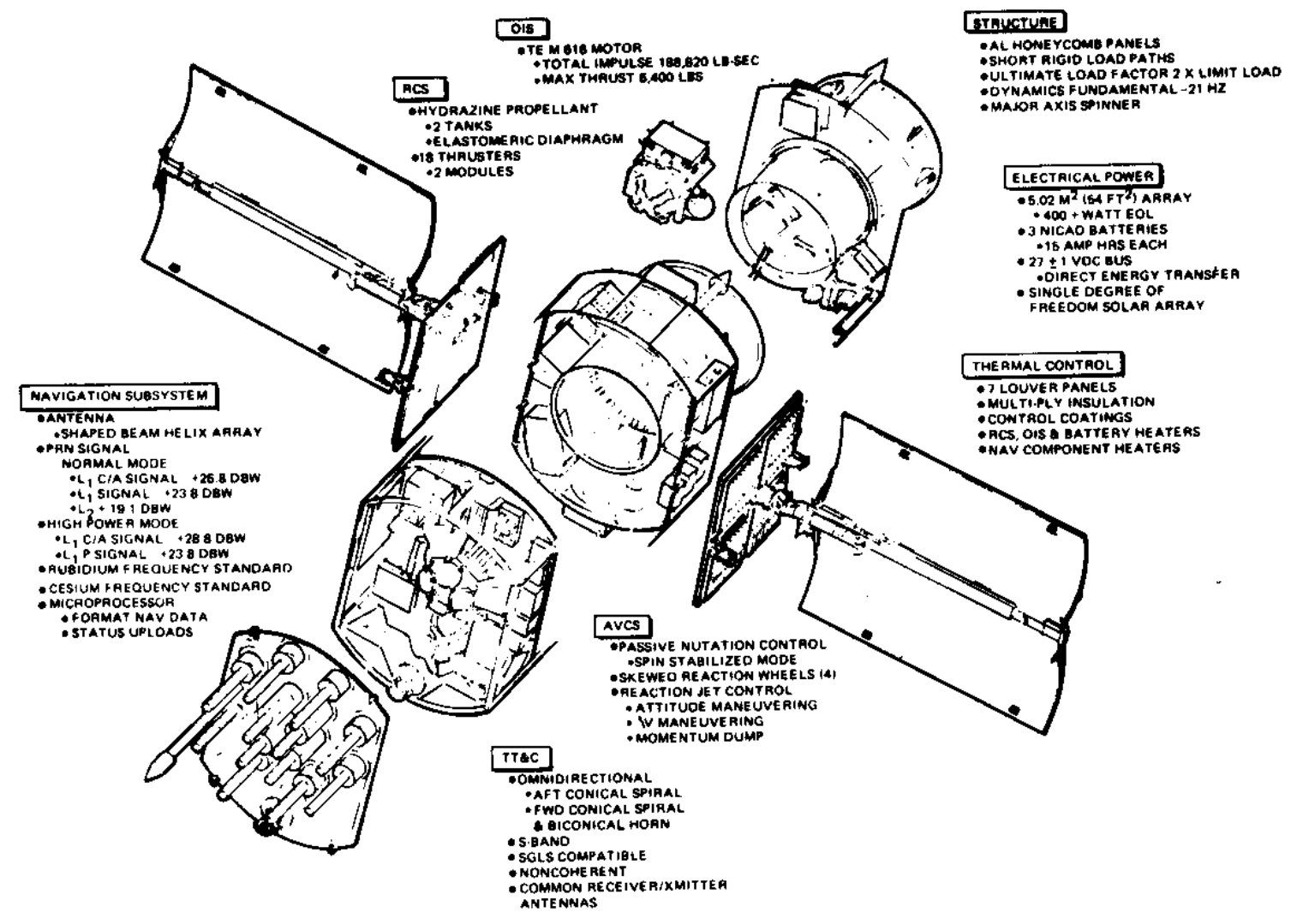

FIGURE 1. GPS SATELLITE

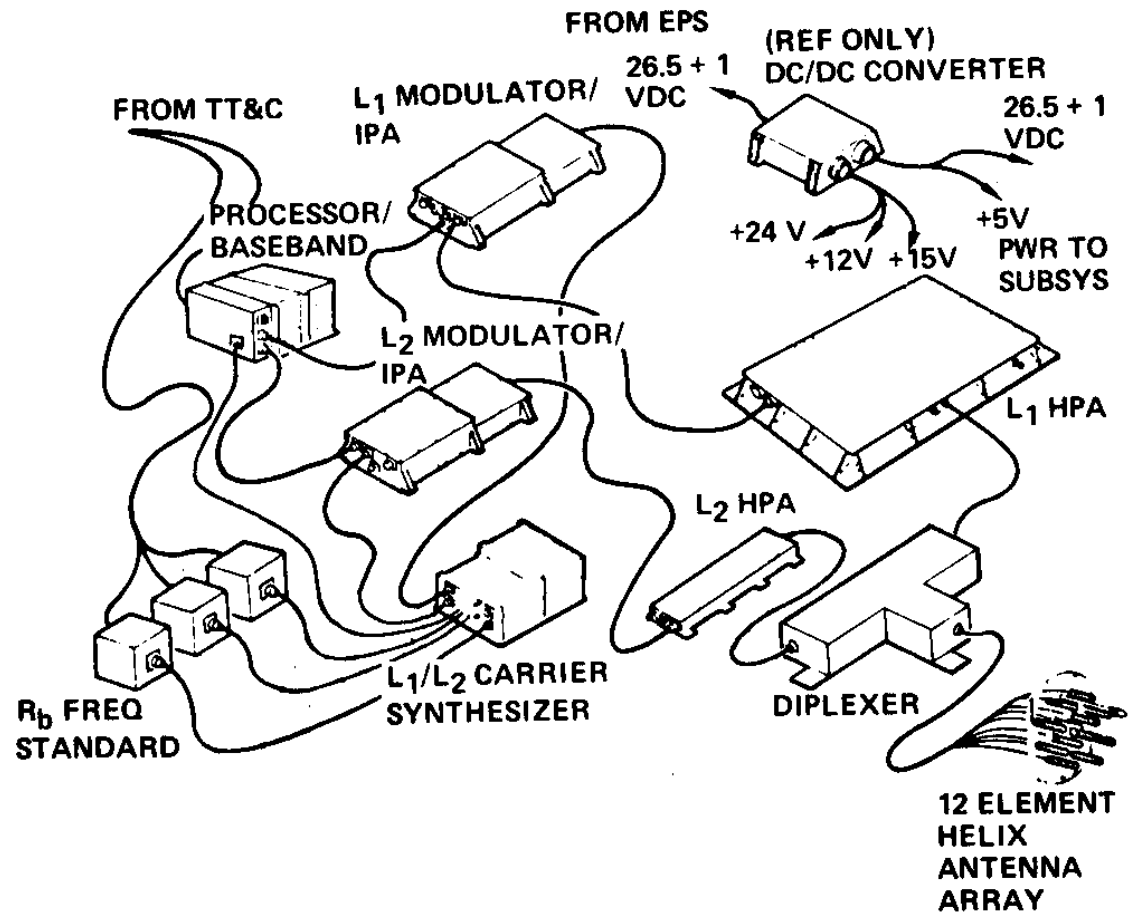

FIGURE 2. GPS NAVIGATION SUBSYSTEM 


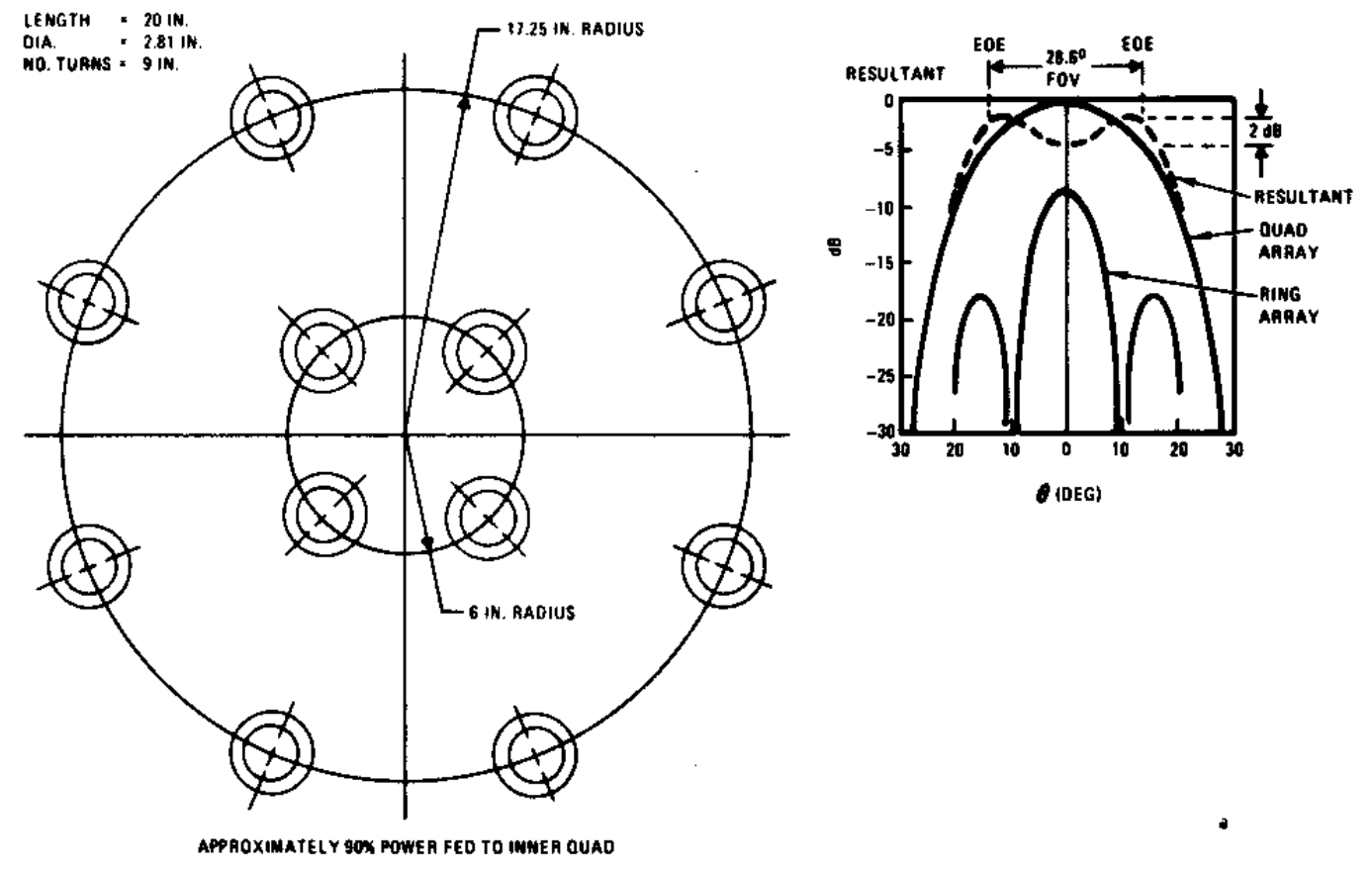

FIGURE 3. GPS NAVIGATION ANTENNA DESIGN

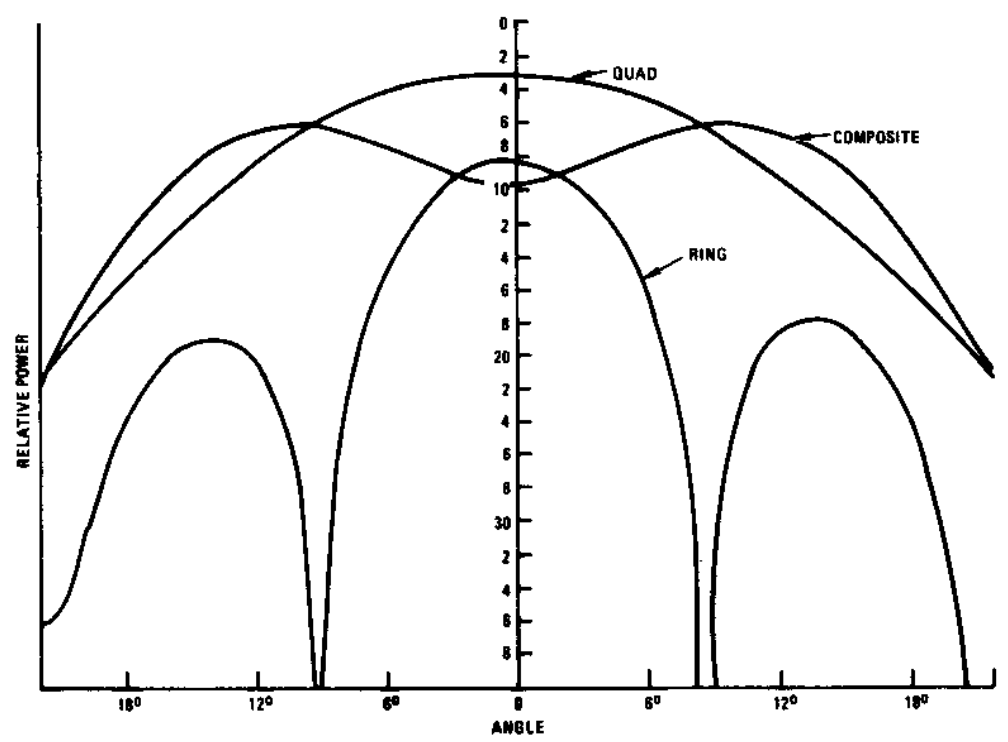

FIGURE 4. MEASURED PATTERNS FOR GPS NAV ANTENNA 

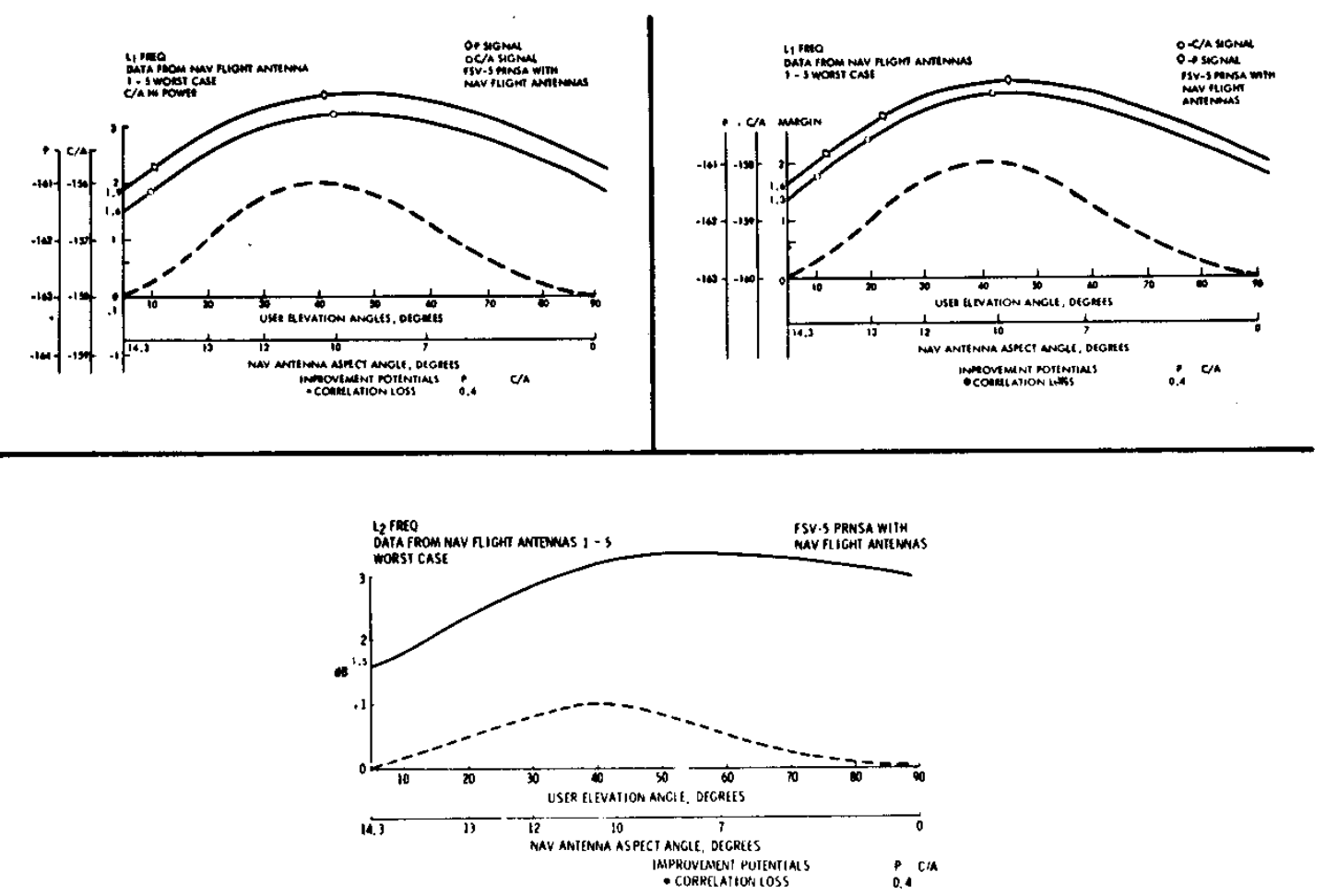

FIGURE 5. NAVIGATION SIGNAL MARGINS

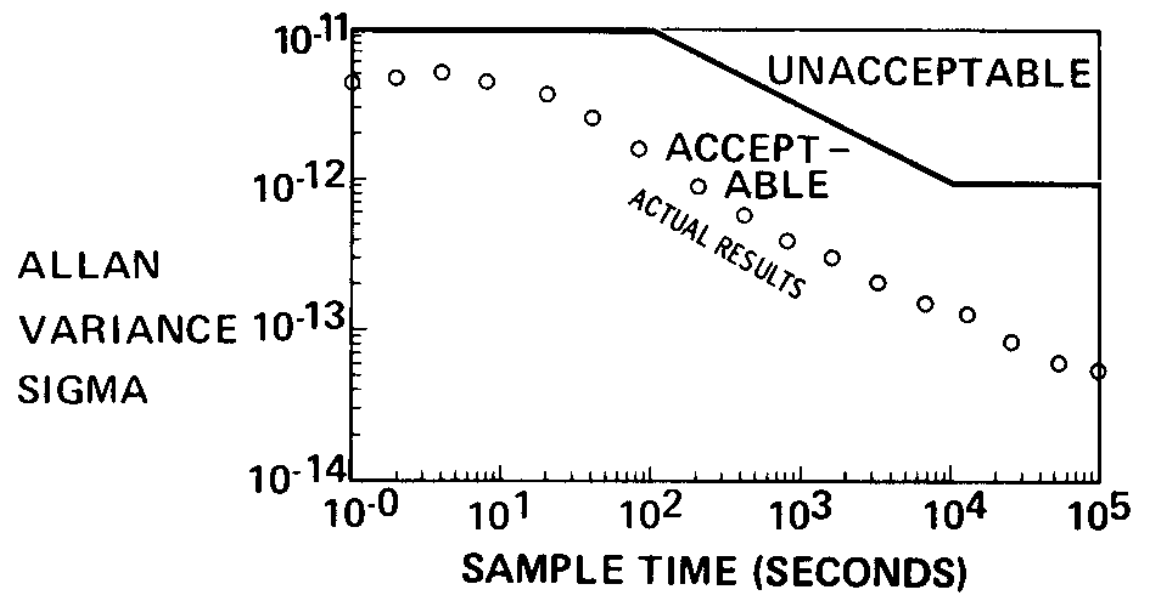

FIGURE 6. FREQUENCY STANDARD STABILITY 


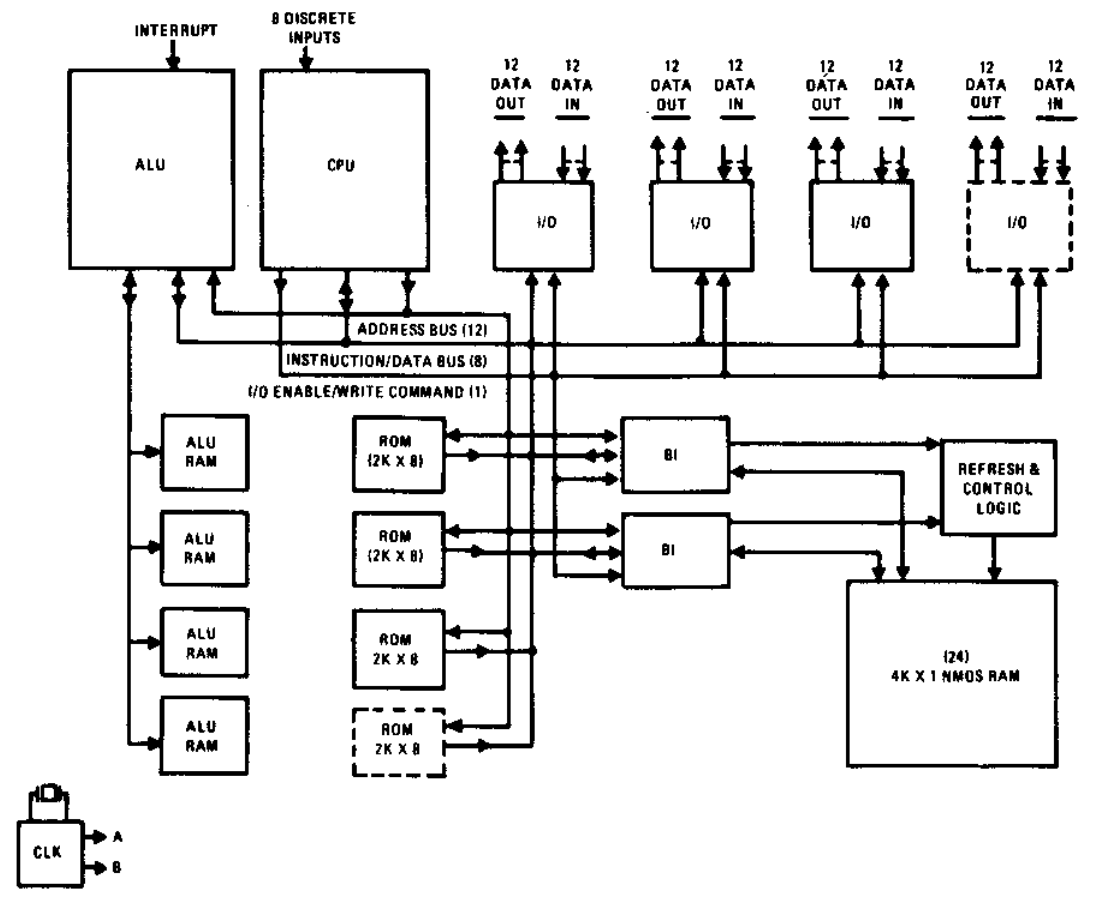

FIGURE 7. NAVIGATION PROCESSOR BLOCK DIAGRAM

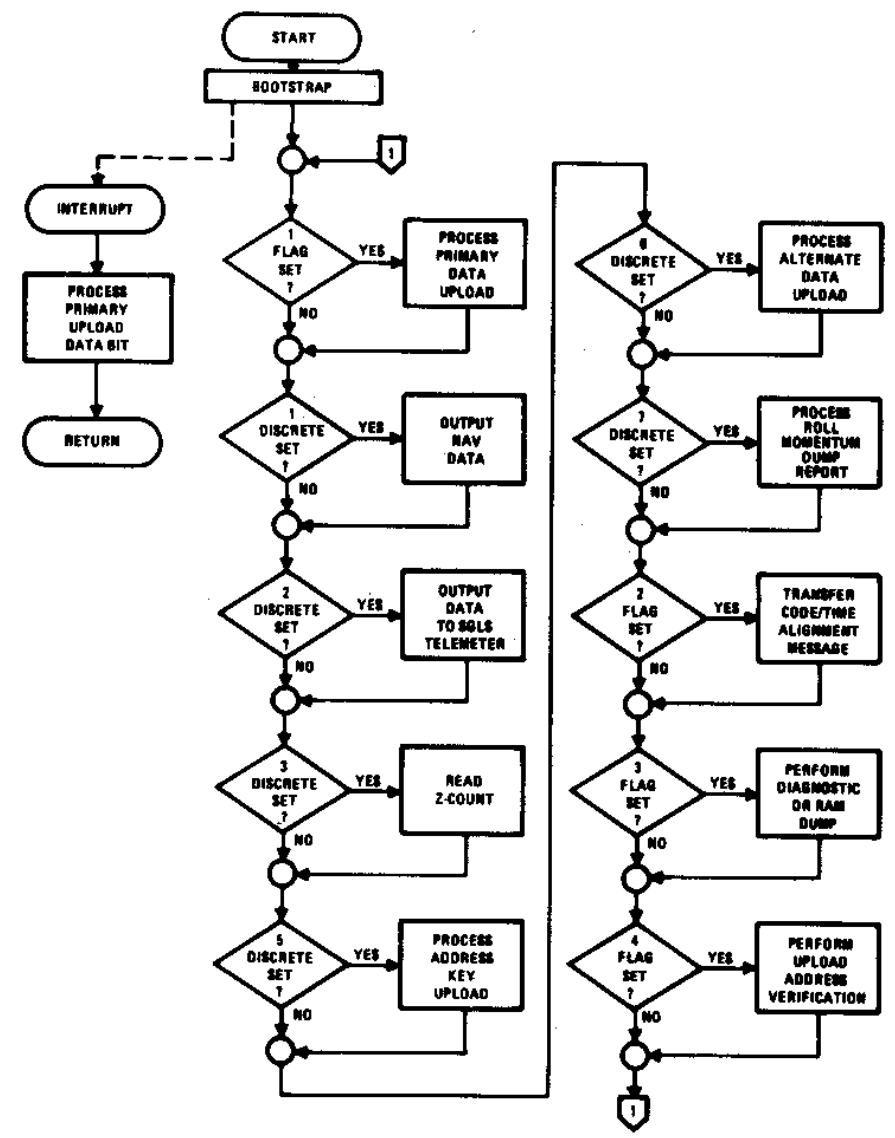

FIGURE 8. GPS ON-BOARD COMPUTER PROGRAM TOP LEVEL FLOW DIAGRAM 


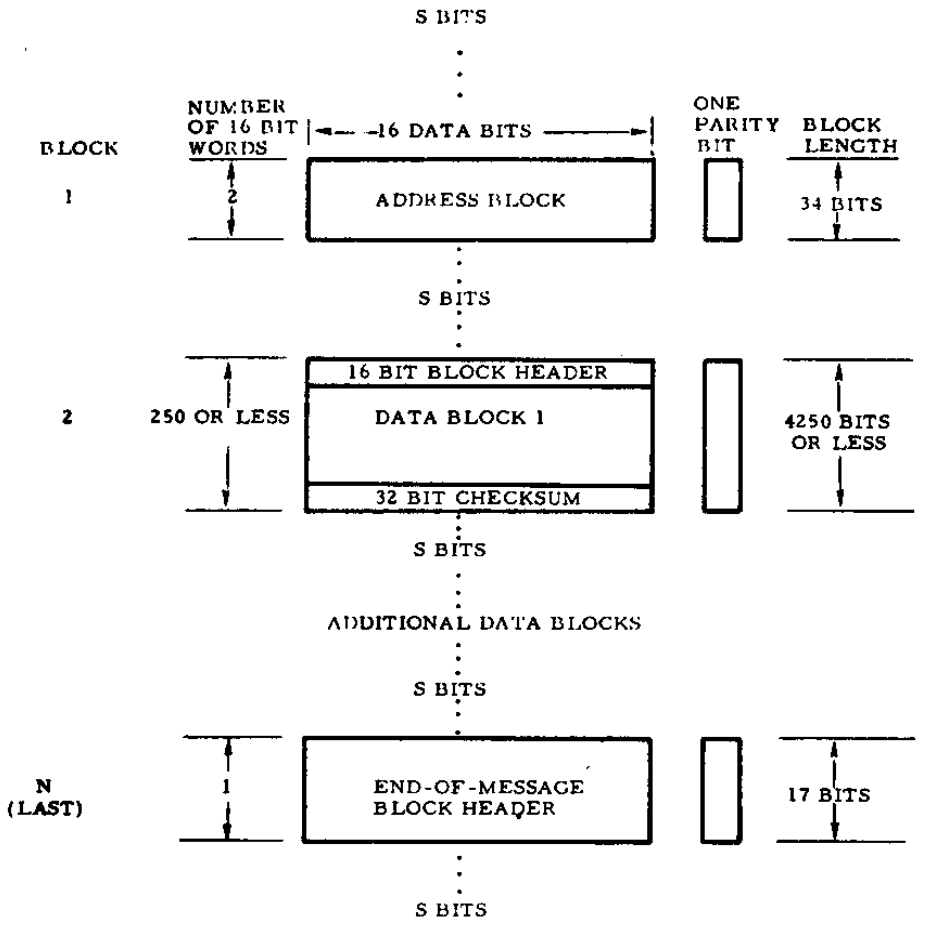

FIGURE 9. PRIMARY UPLOAD MESSAGE FORMAT

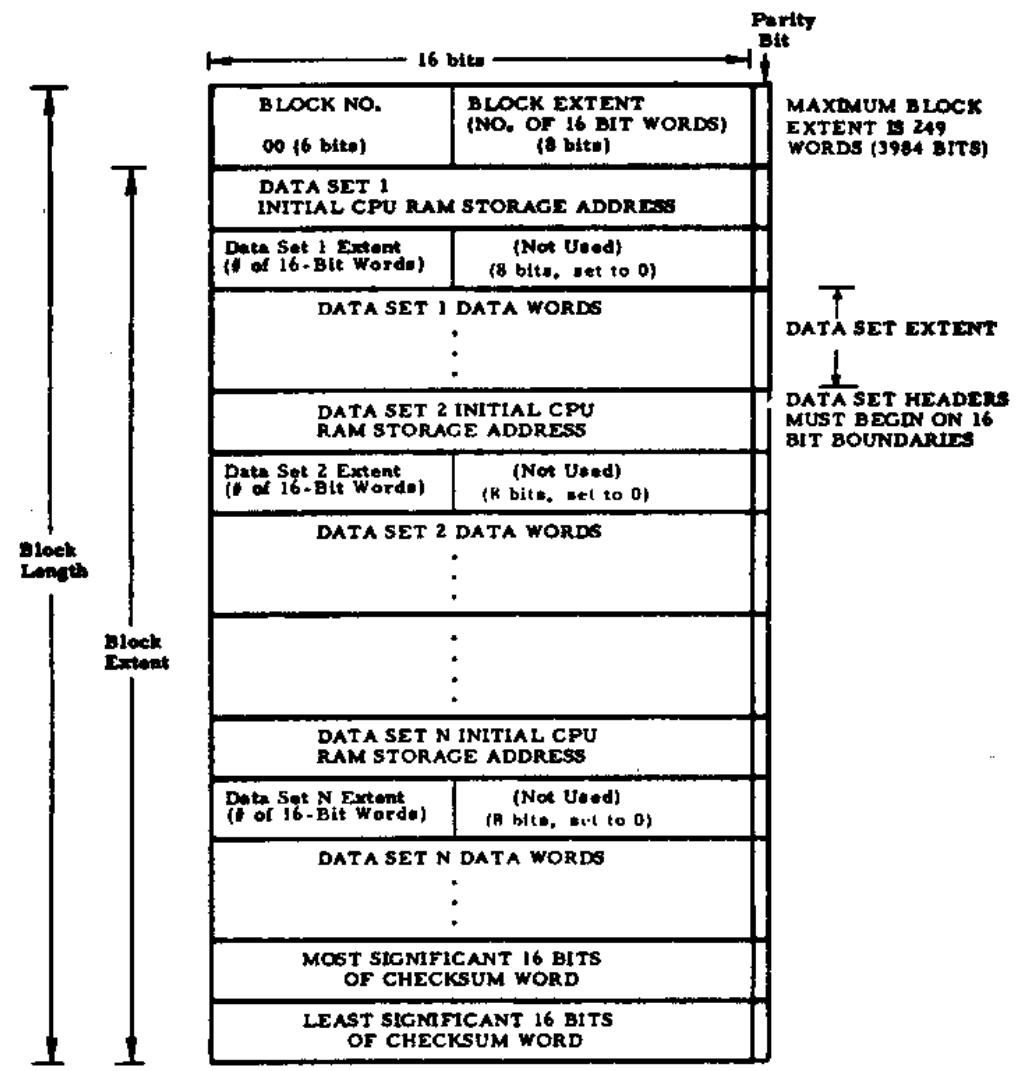

FIGURE 10. PRIMARY UPLOAD BLOCK FORMAT IN UPLOAD BLOCK BUFFER 


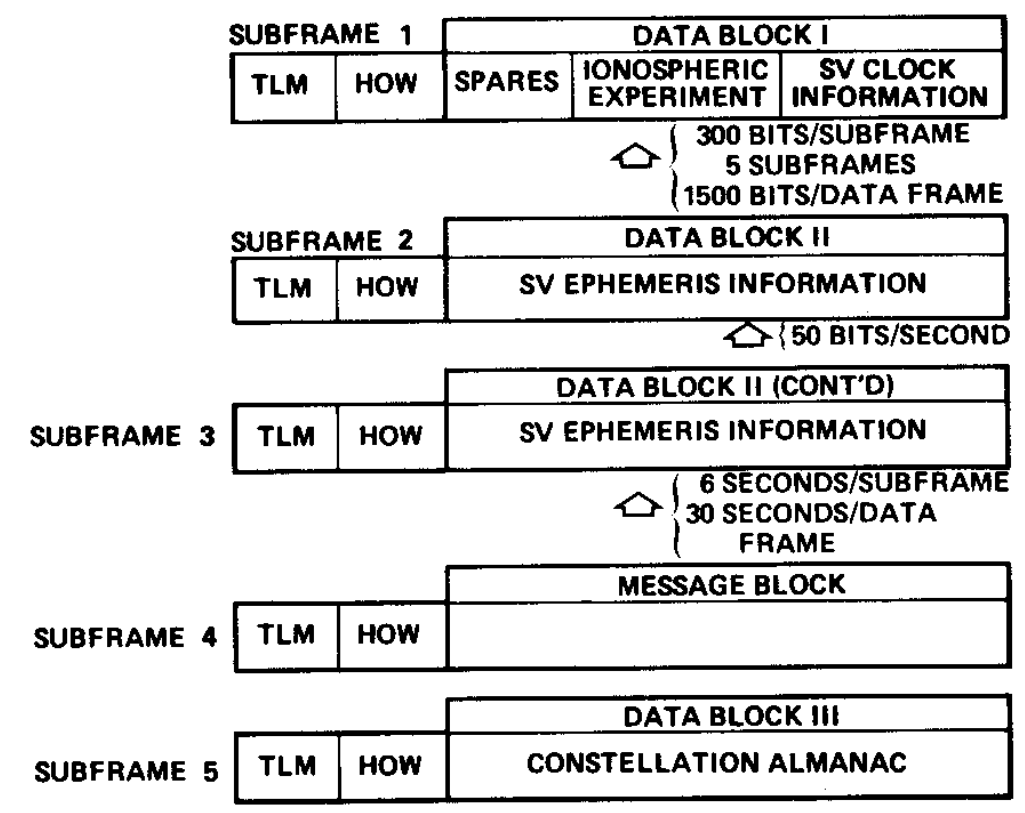

FIGURE 11. EXAMPLE OF NAVIGATION DATA FORMAT 